Separ at or - Based Graph Enbeddi ng i nt o $\mathrm{Hi}$ gher-Di mensi onal Gi ds wi th Snal I Congesti on

\begin{tabular}{|c|c|}
\hline \begin{tabular}{|l} 
著者 \\
\end{tabular} & Nat subayashi Aki ra \\
\hline $\begin{array}{l}\text { j our nal or } \\
\text { publ i cat i on ti tle }\end{array}$ & $\begin{array}{l}\text { Pr oceedi ngs - I EEE I nt er nat i onal Symposi um on } \\
\text { Ci r cui ts and Syst ens }\end{array}$ \\
\hline vol une & 2009 \\
\hline page $r$ ange & $2938-2941$ \\
\hline year & 2009-01-01 \\
\hline URL & ht t p: //hdl . handl e. net /2297/19124 \\
\hline
\end{tabular}




\title{
Separator-Based Graph Embedding into Higher-Dimensional Grids with Small Congestion
}

\author{
Akira Matsubayashi \\ Division of Electrical Engineering and Computer Science, Kanazawa University \\ Kakuma-machi, Kanazawa 920-1192, Japan \\ Email:mbayashi@t.kanazawa-u.ac.jp
}

\begin{abstract}
We study the problem of embedding a guest graph into an optimally-sized grid with minimum edge-congestion. Based on a wellknown notion of graph separator, we prove that any guest graph can be embedded with a smaller edge-congestion as the guest graph has a smaller separator, and as the host grid has a higher dimension. Our results imply the following: An $N$-node planar graph with maximum node degree $\Delta$ can be embedded into an $N$-node $d$-dimensional grid with an edge-congestion of $O\left(\Delta^{2} \log N\right)$ if $d=2, O\left(\Delta^{2} \log \log N\right)$ if $d=3$, and $O\left(\Delta^{2}\right)$ otherwise. An $N$-node graph with maximum node degree $\Delta$ and a treewidth $O(1)$, such as a tree, an outerplanar graph, and a series-parallel graph, can be embedded into an $N$-node $d$-dimensional grid with an edge-congestion of $O(\Delta)$ for $d \geq 2$.
\end{abstract}

\section{INTRODUCTION}

The graph embedding problem is to embed a guest graph into a host graph with certain constraints and/or optimization criteria. The problem has applications such as efficient parallel computation and VLSI layout. The major criteria to measure the efficiency of an embedding are dilation, node-congestion, and edge-congestion.

Graph embedding into grids has been extensively studied so far: An $N$-node complete binary tree can be embedded into an $(N+1)$-node 2 - or 3-dimensional grid with an edge-congestion at most 2 [1][2], into an $(N+1)$-node 4 -dimensional grid with the edge-congestion 1 [1], and into an $(N+O(\sqrt{N}))$-node 2-dimensional grid with the edge-congestion 1. Embedding into 2-dimensional grids in connection with VLSI layout was investigated in, e.g., [3][4][5][6].

Separator-based graph embeddings on hypercubes were presented in [7][8]. It was shown in [8] that an $N$-node graph with maximum node degree $\Delta$ and a node-separator of a polylogarithmic size can be embedded into a $\lceil\log N\rceil$-dimensional cube with a dilation of $O(\log \Delta)$ and an edge-congestion of $O\left(\Delta^{3}\right)$.

A quite general embedding based on the multicommodity flow problem was presented in [9], which showed that an $N$-node bounded degree graph $G$ can be embedded into an $N$-node bounded degree graph $H$ with both dilation and edge-congestion of $O\left(\frac{\log N}{\alpha}\right)$, where $\alpha$ is the flux of $H$. This implies that $G$ can be embedded into an $N$-node $d$-dimensional grid with both dilation and edge-congestion of $O\left(N^{\frac{1}{d}} \log N\right)$.

Any permutation routing on a host graph can be applied to graph embedding. Based on the permutation routing given in [10], we can easily derive that an $N$-node, degree- $\Delta$ graph can be embedded into a $d$-dimensional grid with a dilation of $O\left(d N^{\frac{1}{d}}\right)$ and an edgecongestion of $O\left(\Delta N^{\frac{1}{d}}\right)$.

In this paper, we present a separator-based embedding into optimally-sized grids. This is a generalization of the embedding into 2-dimensional grids presented in [11]. The following are our main theorems:

Theorem 1: An $N$-node, degree- $\Delta$ graph with a $\beta$-node-separator of size $O\left(n^{\alpha}\right)\left(0 \leq \alpha<1, \frac{1}{2} \leq \beta<1\right)$ can be embedded into an $N$-node $d$-dimensional grid $(d \geq 2)$ with a dilation of $O\left(d N^{\frac{1}{d}}\right)$ and an edge-congestion of $O\left(\Delta^{2} \log ^{*} N\right)$ if $\alpha=0$ and $d=2, O\left(d \Delta^{2}+\right.$ $\left.d^{2} \Delta\right)$ if $0 \leq \alpha \leq 1-\frac{2}{d}$ and $d \geq 3, O\left(d \Delta^{2} \log \log N+d^{2} \Delta\right)$ if $1-\frac{2}{d}<\alpha<1-\frac{1}{d}$, and $O\left(d \Delta^{2}\left(N^{\alpha-1+\frac{1}{d}}+\log N\right)+d^{2} \Delta\right)$ if $1-\frac{1}{d} \leq \alpha<1$.

Theorem 2: An $N$-node, degree- $\Delta$ graph with a treewidth $O(1)$ can be embedded into an $N$-node $d$-dimensional grid $(d \geq 2)$ with a dilation of $O\left(d N^{\frac{1}{d}}\right)$ and an edge-congestion of $O\left(d^{2} \Delta\right)$.

We can obtain the following corollaries from the theorems:

Corollary 3: An $N$-node, degree- $\Delta$ planar graph can be embedded into an $N$-node $d$-dimensional grid with an edge-congestion of $O\left(\Delta^{2} \log N\right)$ if $d=2, O\left(\Delta^{2} \log \log N\right)$ if $d=3$, and $O\left(\Delta^{2}\right)$ if $d \geq 4$.

Corollary 4: Any tree, outer planar graph, or series-parallel graph with $N$ nodes and maximum node degree $\Delta$ can be embedded into an $N$-node $d$-dimensional grid with an edge-congestion of $O(\Delta)$ for any $d \geq 2$.

Any embedding has an edge-congestion at least $\frac{\Delta}{2 d}$. Therefore, we achieve an optimal edge-congestion within a constant factor if $\Delta$ and $d \geq 3$ are fixed, and $\alpha \leq 1-\frac{2}{d}$, or if $d$ and the treewidth of the guest graph are fixed.

\section{PRELIMINARIES}

For a non-decreasing function $f(n)=o(n)$, there exists a number $n_{0} \geq 0$ such that $f(n)<n$ for any $n>n_{0}$. Let $n_{f}$ be the minimum integer of such $n_{0}$. We define $f^{*}(n):=\min \left\{c \geq 0 \mid f^{(c)}(n) \leq\right.$ $\left.n_{f}+1\right\}$, where $f^{(0)}(n):=n$ and $f^{(c)}(n):=f\left(f^{(c-1)}(n)\right)$ for an integer $c \geq 1$.

We denote the set of integers $\{i \mid 1 \leq i \leq m\}$ by $[m]$. For a $d$-dimensional vector $v:=\left(x_{i}\right)_{i \in[d]}$, let $\pi_{j}(v):=x_{j}$ and $\bar{\pi}_{j}(v):=\left(x_{i}\right)_{i \in[d] \backslash\{j\}}$ for $j \in[d]$. We use $\pi_{j}$ and $\bar{\pi}_{j}$ also for a set of vectors. E.g., we write $\prod_{i \in[d] \backslash\{j\}}\left[l_{i}\right]$ as $\bar{\pi}_{j}\left(\prod_{i \in[d]}\left[l_{i}\right]\right)$ for positive integers $l_{1}, \ldots, l_{d}$. The $d$-dimensional $l_{1} \times \cdots \times l_{d^{-}}$grid, denoted by $M\left(l_{i}\right)_{i \in[d]}$, is a graph with the node set $\prod_{i \in[d]}\left[l_{i}\right]$ and edge set $\left\{(u, v) \mid \exists j \in[d] \pi_{j}(u)=\pi_{j}(v) \pm 1, \bar{\pi}_{j}(u)=\bar{\pi}_{j}(v)\right\}$. The aspect ratio of $M\left(l_{i}\right)_{i \in[d]}$ is $\max _{i, j \in[d]}\left\{\frac{l_{j}}{l_{i}}\right\}$. An edge $(u, v)$ of $M\left(l_{i}\right)_{i \in[d]}$ with $\pi_{j}(u)=\pi_{j}(v) \pm 1$ is called a dimension- $j$ edge.

A routing request on a graph $H$ is a pair of nodes, a source and target, of $H$. A multiset of routing requests can be represented as a routing graph $R$ with the sources and targets joined by directed edges. It should be noted that $R$ may have parallel edges and loops. In particular, if $H$ is a $d$-dimensional grid, then we define that $\bar{\pi}_{j}(R)$ is a routing graph with the multiset of edges $\left(\bar{\pi}_{j}(u), \bar{\pi}_{j}(v)\right)$ for every $(u, v) \in E(R)$, whereas $\bar{\pi}_{j}(H)$ is simply a $(d-1)$-dimensional grid induced by $\bar{\pi}_{j}(V(H))$. $R$ is called a $p$ - $q$ routing graph, where $p$ and $q$ are the maximum outdegree and indegree of $R$, respectively. A 1-1 routing is also called a permutation routing. We define a routing of $R$ as a mapping $\rho$ that maps $(u, v) \in E(R)$ onto a set of edges of $H$ inducing a path connecting $u$ and $v$. We write $\rho((u, v))$ simply as $\rho(u, v)$. The dilation and edge-congestion of $\rho$ are $\max _{e \in E(R)}|\rho(e)|$ and $\max _{e^{\prime} \in E(H)}\left|\left\{e \in E(R) \mid e^{\prime} \in \rho(e)\right\}\right|$, respectively. 
An embedding of a graph $G$ into a graph $H$ is $\langle\phi, \rho\rangle$ consisting of a one-to-one mapping $\phi: V(G) \rightarrow V(H)$ and a routing $\rho$ of an arbitrary orientation of the graph with node set $\phi(V(G))$ and edge set $\{(\phi(u), \phi(v)) \mid(u, v) \in E(G)\}$. The dilation and edge-congestion of $\langle\phi, \rho\rangle$ are those of $\rho$.

\section{GRAPH SEPARATORS}

The node- and edge-separators are defined as follows: Let $\frac{1}{2} \leq$ $\beta<1$ and $s(n)$ be a non-decreasing function. A graph $G$ has a $\beta$-node(edge, resp.)-separator of size $s(n)$ if we can separate $G$ recursively as follows:

(a) If $|V(G)|=1$, then the separation is done.

(b) For each recursive separation of a subgraph $H$ of $G, H$ can be partitioned by removing at most $s(|V(H)|)$ nodes (edges, resp.) into subgraphs $H_{1}$ and $H_{2}$ such that $\left|V\left(H_{i}\right)\right| \leq \beta|V(H)|+1$ for $i=1,2$.

The author introduced in [11] another scale for edge-separators. Let $x(n)$ be a non-decreasing function. An edge-separator of $G$ has external size $x(n)$ if for each recursive separation of a subgraph $H$ of $G$, the edge-separator partitions $H$ into subgraphs $H_{1}$ and $H_{2}$ such that at most $x\left(\left|V\left(H_{i}\right)\right|\right)$ edges, called external edges, join $V\left(H_{i}\right)$ and $V(G) \backslash V\left(H_{i}\right)$ for $i=1,2$. We will use the following results:

Theorem A: A planar graph has a $\frac{2}{3}$-node-separator of size $O\left(n^{\frac{1}{2}}\right)$ [12].

Lemma $B$ : A degree- $\Delta$ graph $G$ with a $\beta$-node-separator of size $O\left(n^{\alpha}\right)\left(0 \leq \alpha<1, \frac{1}{2} \leq \beta<1\right)$ has a $\frac{1}{2}$-edge-separator of external size $O\left(\Delta^{2} \log n\right)$ if $\alpha=0, O\left(\Delta^{2} n^{\alpha}\right)$ if $0<\alpha \leq 1$ [11].

Lemma $C$ : A degree- $\Delta$ graph with a treewidth $O(1)$ has a $\frac{5}{6}$-edgeseparator of external size $O(\Delta)$ [11]

\section{EMBEDDING ALGORITHM}

\section{A. Permutation Routing and Embedding}

We present lemmas on routing and embedding on grids, which will be used in our main algorithm. The following lemma can easily be obtained from a well-known permutation routing algorithm presented in [10], and hence, we omit the proof.

Lemma 1: Let $R$ be a routing graph on $M:=M\left(l_{i}\right)_{i \in[d]}$ with $l_{h}:=\max _{i \in[d]}\left\{l_{i}\right\} \quad(d \geq 2)$. If $\bar{\pi}_{h}(R)$ is a $p$ - $q$ routing graph on $\bar{\pi}_{h}(M)$, then $R$ can be routed on $M$ with an edge-congestion at $\operatorname{most} 2 \cdot \max \{p, q\}$.

By definition, any routing of a routing graph $R$ is also an embedding of $R$. It is well-known that any undirected graph with degree $\Delta$ has an orientation whose maximum indegree and outdegree are both at most $\left\lceil\frac{\Delta}{2}\right\rceil$. Moreover, such an orientation can be decomposed into $\left\lceil\frac{\Delta}{2}\right\rceil$ edge-disjoint $1-1$ routing graphs by taking $\left\lceil\frac{\Delta}{2}\right\rceil$ matchings of the bipartite graph of sources and targets. Thus, the following holds:

Lemma 2: If any 1-1 routing graph on a graph $H$ can be routed with an edge-congestion at most $c$, then any degree- $\Delta$ graph can be embedded into $H$ with an edge-congestion at most $c\left\lceil\frac{\Delta}{2}\right\rceil$.

\section{B. Separator-Based Embedding}

The following is our core theorem:

Theorem 5: An $N$-node, degree- $\Delta$ graph with a $\beta$-edge-separator $\left(\frac{1}{2} \leq \beta<1\right)$ of external size $x(n)=C n^{\alpha}(0 \leq \alpha<1)$ can be embedded into an $N$-node $d$-dimensional grid $(d \geq 2)$ with a dilation of $O\left(d N^{\frac{1}{d}}\right)$ and an edge-congestion of $O\left(d C f^{*}(N)+d^{2} \Delta\right)$ if $f(n)=o(n), O\left(d C\left(N^{\alpha-1+\frac{1}{d}}+\log N\right)+d^{2} \Delta\right)$ otherwise, where $f(n):=\frac{x^{d}(n)}{n^{d-2}}=C^{d} n^{d(\alpha-1)+2}$.

In fact we can obtain Theorem 1 by combining Lemma B and Theorem 5, and Theorem 2 by Lemma $\mathrm{C}$ and Theorem 5. It is wellknown that any grid is a subgraph of a higher dimensional grid of the same size. Therefore, by combining the fact and Theorems 1 and A, we can obtain Corollary 3. Moreover, Corollary 4 can be obtained by combining Theorem 2 and the fact that trees, outer planar graphs, and series-parallel graphs have treewidth $O(1)$ (e.g., see [13]).

We prove Theorem 5 by constructing a desired embedding algorithm, called SBE.

\section{Definition}

Step 0-Input and Output: For $M:=M\left(l_{i}\right)_{i \in[d]}, k \in[d]$, and an integer $w \geq 1$, let $W_{M}^{k}(w):=\left\{v \in V(M) \mid 2 \leq \pi_{k}(v)<\right.$ $\left.w+2 \leq \pi_{j}(v)<l_{j}-w(j \in[d] \backslash\{k\})\right\}$. For $\{h, k\} \subseteq[d]$, let $S_{M}^{k, h}(w):=\left|\bar{\pi}_{h}\left(W_{M}^{k}(w)\right)\right|=w \prod_{i \in[d] \backslash\{h, k\}}\left(l_{i}-2 w-2\right)$. It should be noted that $S_{M}^{k, h}(w)=S_{M}^{h, k}(w)$ by definition and that it increases as $w$ grows up to $\frac{\min _{i \in[d]}\left\{l_{i}\right\}}{2 d}$. For a mapping $\phi: X \rightarrow Y \subseteq V(M)$, let $\lambda_{h}(\phi):=\max _{v \in \bar{\pi}_{h}(Y)}\left|\left\{s \in X \mid \bar{\pi}_{h}(\phi(s))=v\right\}\right|$.

Algorithm $\operatorname{SBE}(G, X, M, F, k, w)$ :

Input:

- An $N$-node guest graph $G$ with a $\beta$-edge-separator of external size $x(n)=C n^{\alpha}\left(0 \leq \alpha \leq 1, \frac{1}{2} \leq \beta<1\right)$.

- A multiset $X$ of nodes of $G$ incident to distinct external edges of $G$, i.e., a node appears in $X$ as many times as the number of external edges incident to the node.

- A host grid $M=M\left(l_{i}\right)_{i \in[d]}$ with aspect ratio at most $\mu:=$ $\frac{1}{1-\beta}\left(\frac{1}{7 \beta}+e \beta\right)+\frac{5}{4}>4$, where $e$ is base of the natural logarithm, and a set $F \subseteq\left\{v \in V(M) \mid \exists i \in[d] \pi_{i}(v) \in\left\{1, l_{i}\right\}\right\}$ such that $|V(M)|-|F|=N$. Let $l^{\max }:=\max _{i \in[d]}\left\{l_{i}\right\}$ and $l^{\min }:=$ $\min _{i \in[d]}\left\{l_{i}\right\}$.

- A dimension $k \in[d] \backslash\{h\}$ of $M$, where $h \in[d]$ such that $l_{h}=l^{\max }$.

- An integer $1 \leq w \leq \max \left\{\frac{l^{\min }}{2 d}, 1\right\}$.

Output:

- An embedding $\langle\phi, \rho\rangle$ of $G$ into $M$ such that $\phi(V(G)) \cap F=\emptyset$.

- A mapping $\psi: X \rightarrow W_{M}^{k}(w)$ such that $\lambda_{h}(\psi)=\left\lceil\frac{|X|}{S^{k, h}(w)}\right\rceil$.

- A routing $\sigma$ of the routing graph with node set $\phi(X) \cup \psi(X)$ and edge set $\{(\phi(u), \psi(u)) \mid u \in X\}$.

Initially, we arbitrarily choose $F$ and $k$ as desired and perform $\operatorname{SBE}\left(G, \emptyset, M, F, k, w^{\prime}\right)$, where $w^{\prime}$ is $\left\lceil\frac{f^{1 / d}(N)}{\left.4 \mu^{2-1 / d}\right\rceil}\right\rceil$ if $f(n)=o(n)$, $\max \left\{\left\lfloor\frac{l^{\min }}{2 \mu d}\right\rfloor, 1\right\}$ otherwise.

Step 1-Base Embedding: If $f(n)=o(n)$ and $N \leq n_{f}$, or if $l_{h} \leq \max \left\{2 \mu d, 2 \mu\left(\mu+\frac{3}{4}\right)\right\}$, then SBE constructs a base embedding as follows, and quits.

1) If $X=\emptyset$, then let $\phi: V(G) \rightarrow V(M)$ be an arbitrary 1-1 mapping. Otherwise, construct a 1-1 mapping $\phi: V(G) \rightarrow$ $V(M)$ and a mapping $\psi: X \rightarrow W_{M}^{k}(w)$ which minimize the maximum indegree and outdegree of $\bar{\pi}(R)$, where $R$ is the routing graph with node set $\phi(X) \cup \psi(X)$ and edge set $\{(\phi(s), \psi(s)) \mid s \in X\}$.

2) Apply Lemmas 1 and 2 to obtain $\rho$.

3) If $X \neq \emptyset$, then apply Lemma 1 to obtain $\sigma$ of $R$.

Step 2-Separation: Suppose that $G$ is separated with its edgeseparator into two $N_{1}$-node graph $G_{1}$ and $N_{2}$-node $G_{2}$. SBE separates $M$ into two subgrids $M_{1}:=M\left(l_{1}, \ldots, l_{h-1}, m_{1}, \ldots, l_{d}\right)$ and $M_{2}:=M\left(l_{1}, \ldots, l_{h-1}, m_{2}, \ldots, l_{d}\right)$, together with disjoint node sets $F_{1}$ and $F_{2}$ so that $l_{h}=m_{1}+m_{2}-1, F_{1} \cup F_{2}=F \cup\{v \in V(M)$ $\left.\pi_{h}(v)=m_{1}\right\},\left|V\left(M_{i}\right)\right|-\left|F_{i}\right|=N_{i}(i \in[2])$.

Step 3-Resizing Channel:

1) If $w>\max \left\{\frac{l^{\min }}{2 \mu d}, 1\right\}$, then let $\tilde{w}:=\left\lceil\frac{f^{1 / d}(N)}{4 \mu^{2-1 / d} d}\right\rceil$ if $f(n)=$ $o(n)$ and $\tilde{w}:=\max \left\{\left\lfloor\frac{l^{\min }}{2 \mu d}\right\rfloor, 1\right\}$ if $f(n)=\Omega(n)$.

2) If $w \leq \max \left\{\frac{l^{\min }}{2 \mu d}, 1\right\}$, then let $\tilde{w}:=w$. 
Step 4-Recursive Embedding: Let $X_{j}$ be the multiset of nodes of $G_{j}$ incident to distinct external edges of $G_{j}$ for $j \in[2]$. SBE translates the coordinate values of dimension $h$ in the separated subgrids so that a node $v$ with $\pi_{h}(v)=i$ in $M$ has $\pi_{h}(v)=$ $m_{1}-i+1$ in $M_{1}$ if $i \leq m_{1}, \pi_{h}(v)=i-m_{1}+1$ in $M_{2}$ if $i \geq m_{1}$. For each $j \in\{1,2\}$, SBE performs the following procedure:

Case 1: $m_{j} \geq l_{h^{\prime}}$ for $h^{\prime} \in[d] \backslash\{h\}$ with $l_{h^{\prime}}=$ $\max _{i \in[d] \backslash\{h\}}\left\{l_{i}\right\}$. Call $\operatorname{SBE}\left(G_{j}, X_{j}, M_{j}, F_{j}, k, \tilde{w}\right)$. Let $\phi_{j}, \rho_{j}$, $\psi_{j}$, and $\sigma_{j}$ denote the output $\phi, \rho, \psi$, and $\sigma$ of the recursive call, respectively. For consistency with the next case, we define $\tilde{\psi}_{j}:=\psi_{j}$ and $\tilde{\sigma}_{j}\left(\psi_{j}(s), \tilde{\psi}_{j}(s)\right):=\emptyset$ for $s \in X_{j}$.

Case 2: $m_{j}<l_{h^{\prime}}$.

1) $\operatorname{Call~} \operatorname{SBE}\left(G_{j}, X_{j}, M_{j}, F_{j}, h, \tilde{w}\right)$. Let $\phi_{j}, \rho_{j}, \psi_{j}$, and $\sigma_{j}$ denote the output $\phi, \rho, \psi$, and $\sigma$ of the recursive call, respectively.

2) Construct $\tau_{j}: X_{j} \rightarrow W_{M_{j}}^{h}(\tilde{w})$ such that $\lambda_{h^{\prime}}\left(\tau_{j}\right)=\left\lceil\frac{\left|X_{j}\right|}{S_{M_{j}^{h} h^{\prime}(\tilde{w})}}\right\rceil$ and $\lambda_{k}\left(\tau_{j}\right)=\left\lceil\frac{\left|X_{j}\right|}{S_{M_{j}^{h}}^{h, k}(\tilde{w})}\right\rceil$. If $k=h^{\prime}$, then $\tau_{j}$ may be identical with $\psi_{j}$ by induction hypothesis.

3) For each $s \in X_{j}$, let $\tilde{\psi}_{j}(s)$ be the node $v \in V\left(M_{j}\right)$ with $\pi_{k}(v)=\pi_{h}\left(\tau_{j}(s)\right)$ and $\bar{\pi}_{k}(v)=\bar{\pi}_{k}\left(\tau_{j}(s)\right)$.

4) Let $\tilde{\sigma}_{j}$ be a routing from $\psi_{j}\left(X_{j}\right)$ to $\tilde{\psi}_{j}\left(X_{j}\right)$ obtained by concatenating a routing from $\psi_{j}\left(X_{j}\right)$ to $\tau_{j}\left(X_{j}\right)$ on $W_{M_{j}}^{h}(\tilde{w})$ constructed by using Lemma 1 and the dimension- $k$ edges connecting $\tau_{j}(s)$ and $\tilde{\psi}_{j}(s)$ for $s \in X_{j}$.

Step 5-Routing Cut Edges and External Edges:

1) Let $\tilde{\psi}: X \rightarrow W_{M}^{k}(\tilde{w})$ such that $\lambda_{h}(\tilde{\psi})=\left\lceil\frac{|X|}{S_{, h}^{k, h}(\tilde{w})}\right\rceil$.

2) By using Lemma 1 , construct a routing $\tilde{\sigma}$ of the routing graph on $W_{M}^{k}(\tilde{w})$ with node set $\tilde{\psi}_{1}\left(X_{1}\right) \cup \tilde{\psi}_{2}\left(X_{2}\right)$ and edge set $\left\{\left(\tilde{\psi}_{1}\left(s_{1}\right), \tilde{\psi}_{2}\left(s_{2}\right)\right) \mid s_{1} \in X_{1} \backslash X, s_{2} \in X_{2} \backslash X,\left(s_{1}, s_{2}\right) \in\right.$ $E(G)\} \cup \bigcup_{j \in\{1,2\}}\left\{\left(\tilde{\psi}_{j}(s), \tilde{\psi}(s)\right) \mid s \in X_{j} \cap X\right\}$.

3) For $\left(s_{1}, s_{2}\right) \in E(G)$ with $s_{1} \in X_{1} \backslash X$ and $s_{2} \in$ $X_{2} \backslash X$, let $\rho\left(\phi\left(s_{1}\right), \phi\left(s_{2}\right)\right):=\bigcup_{j \in\{1,2\}}\left(\sigma_{j}\left(\phi\left(s_{j}\right), \psi_{j}\left(s_{j}\right)\right) \cup\right.$ $\left.\tilde{\sigma}_{j}\left(\psi_{j}\left(s_{j}\right), \tilde{\psi}_{j}\left(s_{j}\right)\right)\right) \cup \tilde{\sigma}\left(\tilde{\psi}_{1}\left(s_{1}\right), \tilde{\psi}_{2}\left(s_{2}\right)\right)$.

Step 6-Recovering Channel:

Case 1: $\tilde{w}=w$. Let $\psi:=\tilde{\psi}$ and $\sigma(\phi(s), \psi(s)):=$ $\sigma_{j}\left(\phi(s), \psi_{j}(s)\right) \cup \tilde{\sigma}_{j}\left(\psi_{j}(s), \tilde{\psi}_{j}(s)\right) \cup \tilde{\sigma}\left(\tilde{\psi}_{j}(s), \tilde{\psi}(s)\right)$ for $s \in X_{j} \cap X$ $(j \in\{1,2\})$.

Case 2: $\tilde{w}<w$.

1) Let $\chi: X \rightarrow W_{M}^{k}(\tilde{w}) \cap W_{M}^{k}(w)$ such that $\lambda_{h}(\chi)=$ $\left\lceil\frac{|X|}{\left|\bar{\pi}_{h}\left(W_{M}^{k}(\tilde{w}) \cap W_{M}^{k}(w)\right)\right|}\right\rceil$.

2) Let $\psi: X \rightarrow W_{M}^{k}(w)$ such that $\lambda_{h}(\psi)=\left\lceil\frac{|X|}{S_{M}^{k, h}(w)}\right\rceil$.

3) By using Lemma 1 twice, construct a routing $\hat{\sigma}$ from $\tilde{\psi}(X)$ to $\chi(X)$ on $W_{M}^{k}(\tilde{w})$ and a routing $\check{\sigma}$ from $\chi(X)$ to $\psi(X)$ on $W_{M}^{k}(w)$.

4) Let $\sigma(\phi(s), \psi(s))=\sigma_{j}\left(\phi(s), \psi_{j}(s)\right) \cup \tilde{\sigma}_{j}\left(\psi_{j}(s), \tilde{\psi}_{j}(s)\right) \cup$ $\tilde{\sigma}\left(\tilde{\psi}_{j}(s), \tilde{\psi}(s)\right) \cup \hat{\sigma}(\tilde{\psi}(s), \chi(s)) \cup \tilde{\sigma}(\chi(s), \psi(s))$ for $s \in X_{j} \cap X$ $(j \in\{1,2\})$.

\section{Correctness}

It is clear that the base embedding yields a correct output, and that the current procedure yields a correct output on the assumption that the output of its child procedure is correct. We show here that the aspect ratio of $M_{j}(j \in\{1,2\})$ and the range of $\tilde{w}$ fulfill the required conditions. These facts guarantee that a valid input is given to the child procedure.

Lemma 3: For $j \in\{1,2\}, M_{j}$ has an aspect ratio at most $\mu$, and $\min \left\{l_{1}, \ldots, l_{h-1}, m_{j}, \ldots, l_{d}\right\}>2 d$.

Proof: Assume without loss of generally that $m_{1} \leq m_{2}$. Because $M$ has an aspect ratio at most $\mu$ and $l^{\text {min }}>2 d$ (for otherwise, SBE entered the base step), it suffices to prove that $m_{1}>2 d$ and $\frac{l_{h}}{m_{1}} \leq \mu$. Because $N_{j}=\left|M_{j}\right|-\left|F_{j}\right|$ for $j \in\{1,2\}$, it follows that $\left(m_{j}-2\right) \prod_{i \in[d] \backslash\{h\}}\left(l_{i}-2\right) \leq N_{j} \leq m_{j} \prod_{i \in[d] \backslash\{h\}} l_{i}$, and hence, $m_{j}-2 \leq \frac{N_{j}}{\prod_{i \in[d] \backslash\{h\}}\left(l_{i}-2\right)} \leq m_{j} \prod_{i \in[d] \backslash\{h\}} \frac{l_{i}}{l_{i}-2}<$ $\left(\frac{2 d}{2 d-2}\right)^{d-1} m_{j}<e m_{j}$. We have by the inequalities that $\frac{m_{2}-2}{N_{2}}<$ $\frac{e m_{1}}{N_{1}}$. Because $N-N_{1}=N_{2} \leq \beta N+1$, it follows that $N_{1} \geq$ $(1-\beta) N-1 \geq(1-\beta) \frac{N_{2}-1}{\beta}-1=\frac{(1-\beta) N_{2}}{\beta}-\frac{1}{\beta}$. Moreover, it follows that $N \geq \prod_{i \in[d]}\left(l_{i}-2\right) \geq(2 \mu d-2)(2 d-2)^{d-1} \geq 8 \mu-4$, which is larger than $7 \mu$. Hence, it follows that $N_{1} \geq(1-\beta) N-1>$ $7 \mu(1-\beta)-1>7 e \beta$. Thus, we have $m_{2}-2<\frac{e m_{1} N_{2}}{N_{1}} \leq$ $\frac{e m_{1}}{1-\beta}\left(\beta+\frac{1}{N_{1}}\right)<\frac{e m_{1}}{1-\beta}\left(\beta+\frac{1}{7 e \beta}\right) \leq\left(\mu-\frac{5}{4}\right) m_{1}$, which yields $\left(\mu-\frac{1}{4}\right) m_{1}>m_{1}+m_{2}-2=l_{h}-1>2 \mu d-1$. Therefore, it follows that $m_{1}>2 d$ and $\frac{l_{h}}{m_{1}}<\mu-\frac{1}{4}+\frac{1}{m_{1}}<\mu$.

Lemma 4: For $j \in\{1,2\}, 1 \leq \tilde{w} \leq \max \left\{\min \left\{l_{1}, \ldots, l_{h-1}\right.\right.$, $\left.\left.m_{j}, \ldots, l_{d}\right\} /(2 d), 1\right\}$

Proof: It follows from the proof of Lemma 3 that $\frac{m_{j}}{2 d}>$ $\frac{l_{h}}{2 \mu d} \geq \frac{l^{\min }}{2 \mu d}$. Moreover, $\tilde{w} \geq 1$ by definition. Therefore, it suffices to show that $\tilde{w} \leq \max \left\{\frac{l^{\min }}{2 \mu d}, 1\right\}$. This is clear if $f(n)=\Omega(n)$. If $f(n)=o(n)$, then it follows from $N>n_{f}$ that $f^{\frac{1}{d}}(N)<$ $N^{\frac{1}{d}} \leq\left(\prod_{i \in[d]} l_{i}\right)^{\frac{1}{d}} \leq\left(\mu^{d-1}\left(l^{\min }\right)^{d}\right)^{\frac{1}{d}}=\mu^{1-\frac{1}{d}} l^{\text {min }}$, which yields $\tilde{w}=\left\lceil\frac{f^{1 / d}(N)}{4 \mu^{2-1 / d}}\right\rceil \leq\left\lceil\frac{l^{\min }}{4 \mu d}\right\rceil \leq \max \left\{\frac{l^{\min }}{2 \mu d}, 1\right\}$.

\section{Edge-Congestion}

Let $E$ be the set of edges incident to two nodes of $W_{M}^{k}(w)$, and $\tilde{E}$ be the set of edges incident to at least one node of $W_{M}^{k}(\tilde{w})$. Moreover, let $D(N):=\max _{i \in[d]}\left\{\left\lceil\frac{x(N)}{S_{M}^{i, h}(\tilde{w})}\right\rceil\right\}$. It should be noted that $\max _{i \in[d]}\left\{\left\lceil\frac{x(N)}{S_{M}^{i, h}(w)}\right\rceil\right\} \leq D(N)$ for $w \geq \tilde{w}$. For an $n$-node guest graph, in general, we will use $D(n)$ to denote the maximum value of $\left\lceil\frac{x(n)}{S_{H}^{i, j}(\tilde{w})}\right\rceil$ over all feasible $d$-dimensional host grids $H,\{i, j\} \subseteq[d]$, and $\tilde{w}$.

Lemma 5: The routings $\rho$ and $\sigma$ impose an edge-congestion of $O(D(N))$ on edges of $E \cup \tilde{E}$ and $O\left(D\left(N_{j}\right)\right)$ on edges incident to two nodes of $W_{M_{j}}^{h}(\tilde{w})$.

Proof: By the definition of SBE, it follows that $\lambda_{h^{\prime}}\left(\psi_{j}\right)=$ $\lambda_{h^{\prime}}\left(\tau_{j}\right) \leq\left\lceil\frac{x\left(N_{j}\right)}{S_{M_{j}}^{h, h^{\prime}}(\tilde{w})}\right\rceil=\lambda_{k}\left(\tau_{j}\right)=\lambda_{h}\left(\tilde{\psi}_{j}\right)=D\left(N_{j}\right) \leq$ $\left\lceil\frac{x(N)}{S_{M}^{h, h^{\prime}}(\tilde{w})}\right\rceil=D(N)$. Moreover, it follows that $\lambda_{h}(\psi) \leq$ $\lambda_{h}(\tilde{\psi}) \leq \lambda_{h}(\chi)=\left\lceil\frac{|X|}{\tilde{w} \prod_{i \in[d] \backslash\{h, k\}}\left(l_{i}-2 w-2\right)}\right\rceil=\left\lceil\left(\prod_{i \in[d] \backslash\{h, k\}}\right.\right.$ $\left.\left.\frac{l_{i}-2 \tilde{w}-2}{l_{i}-2 w-2}\right) \frac{|X|}{S_{M}^{k, h}(\tilde{w})}\right\rceil$, which is at most $\left\lceil\frac{e x(N)}{S_{M}^{k, h}(\tilde{w})}\right\rceil \leq 3 D(N)$ because $\prod_{i \in[d] \backslash\{h, k\}} \frac{l_{i}-2 \tilde{w}-2}{l_{i}-2 w-2}<\prod_{i \in[d] \backslash\{h, k\}} \frac{l_{i}-2}{l_{i}-l^{\min } / d-2}<\left(\frac{2 d-2}{2 d-4}\right)^{d-2}<$ $e$. Therefore, the lemma holds by Lemma 1 .

Lemma 6: If $l^{\mathrm{min}}>2 d$, then $D(N)$ is at most $O(C)$ if $\alpha \leq 1-\frac{2}{d}$, $O(d)$ if $1-\frac{2}{d}<\alpha<1-\frac{1}{d}$, and $O\left(d C N^{\alpha-1+\frac{1}{d}}\right)$ if $\alpha \geq 1-\frac{1}{d}$.

Proof: It follows that $N \geq \prod_{i \in[d]}\left(l_{i}-2\right)>(1-$ $\left.\frac{1}{d}\right)^{d} \prod_{i \in[d]} l_{i} \geq \frac{1}{4} l_{h} l_{k}\left(\frac{l_{h} l_{k}}{\mu^{2}}\right)^{\frac{d-1}{2}}=\frac{1}{4} \mu^{-(d-2)}\left(l_{h} l_{k}\right)^{\frac{d}{2}}$, which yields $l_{h} l_{k}<4 \mu^{2-\frac{4}{d}} N^{\frac{2}{d}}$. Because $\tilde{w} \leq w \leq \frac{l^{\min }}{2 d}$, it follows that $\prod_{i \in[d] \backslash\{h, k\}}\left(l_{i}-2 \tilde{w}-2\right) \geq \prod_{i \in[d] \backslash\{h, k\}}\left(l_{i}-\frac{l^{\min }}{d}-2\right)>(1-$ $\left.\frac{2}{d}\right)^{d-2} \prod_{i \in[d] \backslash\{h, k\}} l_{i}>\frac{\prod_{i \in[d]} l_{i}}{e^{2} l_{h} l_{k}}>\frac{N^{1-2 / d}}{4 e^{2} \mu^{2-4 / d}}$. Therefore, $D(N) \leq$ $\left\lceil\frac{4 e^{2} \mu^{2-4 / d} C N^{\alpha-1+2 / d}}{\tilde{w}}\right\rceil$.

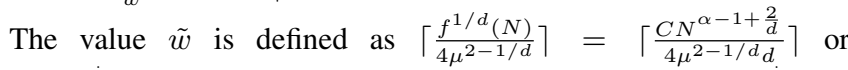
$\max \left\{\left\lfloor\frac{l^{\min }}{2 \mu d}\right\rfloor, 1\right\}$ in the current procedure if $w>\max \left\{\frac{l^{\min }}{2 \mu d}, 1\right\}$. Otherwise, $\tilde{w}$ has been determined similarly in an ancestor procedure, where the host graph and the shortest side length of the host grid are larger than or equal to those in the current procedure. Thus, we have 
$\tilde{w} \geq \frac{C N^{\alpha-1+\frac{2}{d}}}{4 \mu^{2-1 / d} d}$ if $f(n)=o(n), \tilde{w} \geq \max \left\{\left\lfloor\frac{l^{\min }}{2 \mu d}\right\rfloor, 1\right\}$ otherwise. If $\alpha \leq 1-\frac{2}{d}$, then the lemma holds by $\tilde{w} \geq 1$ and by $N^{\alpha-1+\frac{2}{d}} \leq 1$. If $1-\frac{2}{d}<\alpha<1-\frac{1}{d}$, then the lemma holds by $\tilde{w} \geq \frac{C N^{\alpha-1+\frac{2}{d}}}{4 \mu^{2-1 / d} d}$. If $\alpha \geq 1-\frac{1}{d}$, then $\tilde{w} \geq \max \left\{\left\lfloor\frac{l^{\mathrm{min}}}{2 \mu d}\right\rfloor, 1\right\} \geq \frac{l^{\mathrm{min}}}{4 \mu d}$, which is at least $\frac{N^{1 / d}}{4 \mu^{2-1 / d}}$ because $N^{\frac{1}{d}} \leq\left(\prod_{i \in[d]} l_{i}\right)^{\frac{1}{d}} \leq \mu^{1-\frac{1}{d}} l^{\mathrm{min}}$.

Lemma 7: The edge-congestion $B$ of the base embedding is at most $O(d \Delta+C)$.

Proof: The edge-congestion of $\langle\phi, \rho\rangle$ is at most $2\left\lceil\frac{\Delta}{2}\right\rceil l_{h}$ by Lemmas 1 and 2. If $X \neq \emptyset$, then the maximum outdegree $p$ and indegree $q$ of $\bar{\pi}(R)$ overall nodes of $\phi(X)$ and $\phi\left(X^{\prime}\right)$, respectively, satisfy $p \leq \frac{|X|}{\prod_{i \in[d] \backslash\{h\}}\left(l_{i}-2\right)}+\Delta \leq D(N)+\Delta$ and $q=\lambda_{h}(\psi)=$ $\left\lceil\frac{|X|}{S_{M}^{k, h}(w)}\right\rceil \leq D(N)$. Because $X \neq \emptyset$ only if the base embedding is called by a parent procedure, $l^{\min }>2 d$ by Lemma 3 . Therefore, by Lemmas 1 and 6 , we have $B \leq 2\left(\left\lceil\frac{\Delta}{2}\right\rceil l_{h}+\max \{p, q\}\right)=$ $O\left(l_{h} \Delta+D(N)\right)$, which is $O(d \Delta+C)$ because $N^{\frac{1}{d}} \leq l_{h}=O(d)$ and $D(N)=O\left(d C N^{\alpha-1+\frac{1}{d}}\right)=O\left(C d^{-d(1-\alpha)+2}\right)=\bar{O}(C)$.

We now estimate the total congestion of an edge $r$ of $M$. In what follows, $M_{1}:=M, \ldots, M_{z}$ denote a maximal sequence of subgrids of $M$ containing $r$ such that $M_{j+1}(1 \leq j<z)$ is one of the two subgrids into which $M_{j}$ is separated in the $j$ th recursive call of SBE. I.e., we suppose that base embedding is performed in the $z$ th call. We divide the sequence of recursive calls into phases such that each call setting $\tilde{w}<w$ in Step 3 begins a new phase. Let $n_{j}$ be the number of nodes of the guest graph to be embedded into $M_{j}$. Other symbols with an additional subscript, such as $h_{j}, k_{j}, w_{j}, E_{j}$, and $l_{i, j}(i \in[d])$ denote the corresponding parameters in the $j$ th recursive call. We denote $W_{M_{j}}^{k_{j}}\left(w_{j}\right)$ and $W_{M_{j}}^{k_{j}}\left(\tilde{w}_{j}\right)$ by $W_{j}$ and $\tilde{W}_{j}$, respectively. Recall that $\tilde{w}_{j}=w_{j+1}$ for $j \in[z-1]$, and that if the $j$ th call does not begin a phase, then $w_{j}=\tilde{w}_{j}$, and hence, $W_{j}=\tilde{W}_{j}$.

Lemma 8: Suppose that the $a$ th and $b$ th recursive calls are the first calls of consecutive phases. If the $j$ th recursive call $(a \leq j<b)$ performs a recursive embedding into $M_{j+1}$ of Case 2 in Step 4, then $\tilde{W}_{c} \cap W_{y}=\emptyset$ for $a \leq c \leq j<y \leq b$.

Proof: $W_{j+1}$ is a set of inner nodes of $M_{j}$, i.e., $v \in W_{j+1}$ has $\tilde{w}_{j}+2 \leq \pi_{i}(v)<l_{i, j}-\tilde{w}_{j}$ in $M_{j}$ for $i \in[d]$. By $\tilde{w}_{c}=w_{j}$, inner nodes of $M_{j}$ are also inner nodes in $M_{c}$. Moreover, since any $v \in \tilde{W}_{c}$ has $\pi_{k_{c}}(v) \leq \tilde{w}_{c}+1$ in $M_{c}$, and since $W_{y}$ is either a subset of $W_{j+1}$ or a set of inner nodes of $M_{j+1}$, we have $v \notin W_{y}$.

Lemma 9: Suppose that the $a$ th and $b$ th recursive calls are the first calls of consecutive phases. The number $Q$ of sets in $\tilde{E}_{a}, \tilde{E}_{a+1}, \ldots$, $\tilde{E}_{b-1}$, and $E_{b}$ that contain $r$ is at most $\left\lceil\left(\mu-\frac{3}{4}\right) \ln (2 \mu-1)\right\rceil=O(1)$.

Proof: By definition, if $\tilde{W}_{c} \cap W_{y}=\emptyset$ for $a \leq c<y \leq$ $b$, then $\tilde{E}_{c} \cap E_{y}=\emptyset$. Thus, by Lemma 8 , it suffices to estimate the maximum number of consecutive recursive calls which have the same $h$. It should be noted that the condition of Case 1 in Step 4 implies the same $h$ between the consecutive recursive calls. Let $h:=$ $h_{c}=h_{c+1}=\cdots=h_{j} \neq h_{j+1}$. It follows from the proof of Lemma 3 that for $c \leq i<j, l_{h, i+1} \leq \max \left\{m_{1, i}, m_{2, i}\right\}=l_{h, i}-$ $\min \left\{m_{1, i}, m_{2, i}\right\}+1<l_{h, i}-\frac{l_{h, i}-1}{\mu-1 / 4}+1=\left(1-\frac{1}{\mu-1 / 4}\right) l_{h, i}+(1+$ $\left.\frac{1}{\mu-1 / 4}\right)$, which yields $l_{h, j}<\left(1-\frac{1}{\mu-1 / 4}\right)^{j-c}\left(l_{h, c}-\mu-\frac{3}{4}\right)+\mu+\frac{3}{4}$. Because $l_{h, j} \geq l_{c}^{\min } \geq \frac{l_{h, c}}{\mu}$, we have $l_{h, j}<\left(1-\frac{1}{\mu-1 / 4}\right)^{j-c}\left(\mu l_{h, j}-\right.$ $\left.\mu-\frac{3}{4}\right)+\mu+\frac{3}{4}$. Because $l_{h, j} \geq l_{a}^{\min } \geq \frac{l_{h, a}}{\mu}>2\left(\mu+\frac{3}{4}\right)$, it follows that $\left(1+\frac{1}{\mu-5 / 4}\right)^{j-c}<\frac{\mu l_{h, j}-\mu-3 / 4}{l_{h, j}-\mu-3 / 4}<2 \mu-1=e^{\ln (2 \mu-1)}<$ $\left(1+\frac{1}{\mu-5 / 4}\right)^{(\mu-3 / 4) \ln (2 \mu-1)}$. Therefore, we have $Q=j-c+1 \leq$ $\left\lceil\left(\mu-\frac{3}{4}\right) \ln (2 \mu-1)\right\rceil$.

Lemma 10: The number $P$ of phases is at $\operatorname{most} \max \left\{f^{*}(N), 1\right\}$ if $f(n)=o(n), \max \left\{\log _{\beta^{-1}} N, 1\right\}$ otherwise.
Proof: Assume $P \geq 2$. We first consider the case of $f(n)=$ $\Omega(n)$. Because $n_{i} \leq \beta n_{i-1}+1$ for $2 \leq i \leq z$, it follows that $n_{z-1} \leq \beta^{z-2}\left(N-\frac{1}{1-\beta}\right)+\frac{1}{1-\beta}<\beta^{z-2} N+\frac{1}{1-\beta}$. Thus, we have $z<\log _{\beta^{-1}} N-\log _{\beta^{-1}}\left\{\left(n_{z-1}-\frac{1}{1-\beta}\right) \beta^{2}\right\}$. Because $n_{z-1}>l_{z-1}^{\max }>$ $2 \mu d>2 \mu$, it follows that $\left(n_{z-1}-\frac{1}{1-\beta}\right) \beta^{2}>\left(2 \mu-\frac{1}{1-\beta}\right) \beta^{2}>$ $\mu \beta^{2}>1$. Therefore, $P \leq z<\log _{\beta^{-1}} N$.

We next consider the case of $f(n)=o(n)$. Suppose that the $a$ th and $b$ th recursive calls $(a<b)$ are the first calls of consecutive phases. Because $n_{b}^{\frac{1}{d}} \leq \mu^{1-\frac{1}{d}} l_{b}^{\min }$ as shown in the proof of Lemma 6 , it follows that $\tilde{w}_{a}=w_{b}>\frac{l_{b}^{\min }}{2 \mu d} \geq \frac{n_{b}^{1 / d}}{2 \mu^{2-1 / d}}$. Moreover, because the $a$ th call is not in the last phase, we have $2 \leq \tilde{w}_{a}=\left\lceil\frac{f^{1 / d}\left(n_{a}\right)}{\left.4 \mu^{2-1 / d}\right\rceil}\right\rfloor$ $\frac{f^{1 / d}\left(n_{a}\right)}{2 \mu^{2-1 / d} d}$. Therefore, $f\left(n_{a}\right)>n_{b}$. By $b<z$, we have $f^{(P-1)}(N)>$ $n_{z-1} \geq n_{f}+1$, which means $P-1<f^{*}(N)$.

Lemma 11: The edge-congestion on $r$ is at most $O\left(d C f^{*}(N)+\right.$ $\left.d^{2} \Delta\right)$ if $f(n)=o(n), O\left(d C\left(N^{\alpha-1+\frac{1}{d}}+\log N\right)+d^{2} \Delta\right)$ otherwise.

Proof: The edge $r$ is congested in recursive calls and the base embedding on $M_{z}$. Moreover, if $r$ joins two nodes on the boundary of $M_{z}$, then there can exist at most $2(d-1)$ grids containing $r$ on which base embeddings are constructed. Thus, the edge-congestion on $r$ is at most $\sum_{i=1}^{P} O\left(D\left(n_{a_{i}}\right)\right) \cdot Q+2(d-1) B$ by Lemma 5 , where $a_{i}$ is the number $j$ maximizing $D\left(n_{j}\right)$ in the $i$ th phase.

If $f(n)=o(n)$, then the edge-congestion is at most $O((d+$ $\left.C)\left(f^{*}(N)+1\right)+d(d \Delta+C)\right)=O\left(d C f^{*}(N)+d^{2} \Delta\right)$ by Lemmas 6, 7, 9, and 10. Assume $f(n)=\Omega(n)$. As shown in the proof of Lemma 10 , we have $n_{j} \leq \beta^{j-1} N+\frac{1}{\beta-1}$ for $j \in[z]$. Thus, it follows from Lemmas 6 and 10 that $\sum_{i=1}^{P} D\left(n_{a_{i}}\right) \leq \sum_{j=1}^{z} D\left(n_{j}\right) \leq$ $\sum_{j=1}^{\log _{1 / \beta} N} O\left(d C\left(\beta^{j-1} N+\frac{1}{\beta-1}\right)^{\alpha-1+\frac{1}{d}}\right)=O\left(d C\left(N^{\alpha-1+\frac{1}{d}}+\right.\right.$ $\log N))$. Therefore, by combining the inequality and Lemmas 7 and 9 , the edge-congestion on $r$ is at most $O\left(d C\left(N^{\alpha-1+\frac{1}{d}}+\right.\right.$ $\log N)+d(d \Delta+C))=O\left(d C\left(N^{\alpha-1+\frac{1}{d}}+\log N\right)+d^{2} \Delta\right)$.

The dilation of SBE is $O\left(\sum_{j=1}^{z} d n_{j}^{\frac{1}{d}}\right)=O\left(d \sum_{j=1}^{z}\left(\beta^{j-1} N+\right.\right.$ $\left.\left.\frac{1}{\beta-1}\right)^{\frac{1}{d}}\right)=O\left(d N^{\frac{1}{d}}\right)$. Therefore, we have obtained Theorem 5 .

\section{REFERENCES}

[1] S.-K. Lee and H.-A. Choi, "Link-disjoint embedding of complete binary trees in meshes," Networks, vol. 30, no. 4, pp. 283-292, 1997.

[2] P. Zienicke, "Embeddings of treelike graphs into 2-dimensional meshes," in Lecture Notes in Computer Science. Springer-Verlag, 1991, vol. 484, pp. 182-192.

[3] S. N. Bhatt and F. T. Leighton, "A framework for solving VLSI graph layout problems," J. Comput. Syst. Sci., vol. 28, pp. 300-343, 1984.

[4] C. E. Leiserson, Area Efficient VLSI Computation. MIT Press, 1983.

[5] A. Matsubayashi, "VLSI layout of trees into grids of minimum width," IEICE Trans. Fundamentals, vol. E87-A, no. 5, pp. 1059-1069, 2004.

[6] L. G. Valiant, "Universality considerations in VLSI circuits," IEEE Trans. Comput., vol. C-30, no. 2, pp. 135-140, 1981.

[7] S. N. Bhatt, F. R. K. Chung, F. T. Leighton, and A. L. Rosenberg, "Efficient embeddings of trees in hypercubes," SIAM J. Comput., vol. 21, no. 1, pp. 151-162, 1992.

[8] V. Heun and E. W. Mayr, "Efficient embeddings into hypercube-like topologies," The Computer Journal, vol. 46, pp. 632-644, 2003.

[9] T. Leighton and S. Rao, "Multicommodity max-flow min-cut theorems and their use in designing approximation algorithms," J. ACM, vol. 46, no. 6, pp. 787-832, 1999.

[10] M. Baumslag and F. S. Annexstein, "A unified framework for off-line permutation routing in parallel networks." Mathematical Systems Theory, vol. 24, no. 4, pp. 233-251, 1991.

[11] A. Matsubayashi, "Small congestion embedding of separable graphs into grids of the same size," in Proc. 2005 IEEE Int. Symp. Circuits and Systems, 2005, pp. 1354-1357.

[12] R. J. Lipton and R. E. Tarjan, "A separator theorem for planar graphs," SIAM J. Appl. Math., vol. 36, no. 2, pp. 177-189, 1979.

[13] H. L. Bodlaender, "A tourist guide through treewidth," Acta Cybern., vol. 11 , no. $1-2$, pp. 1-22, 1993. 